\title{
Wetting, adhesion and droplet impact on face masks
}

\author{
Kiran Raj Melayil and Sushanta K. Mitra* \\ Micro \& Nano-scale Transport Laboratory, Waterloo Institute for Nanotechnology, Department of Mechanical and Mechatronics \\ Engineering, University of Waterloo, 200 University Avenue West, Waterloo, Ontario N2L 3G1, Canada \\ * Corresponding author: skmitra@uwaterloo.ca
}

\begin{abstract}
In the present pandemic time, face masks are found to be the most effective strategy against the spread of the virus within the community. As aerosol-based spreading of the virus is considered as the primary mode of transmission, the interaction of masks with incoming droplets needs to be understood thoroughly for an effective usage among the public. In the present work, we explore the interactions of the droplets over the most commonly used 3-ply surgical masks. A detailed study of the wetting signature, adhesion and impact dynamics of water droplets and microbe-laden droplets is carried out for both sides of the mask. We found that the interfacial characteristics of the incoming droplets with the mask are very similar for the front and the back side of the mask. Further, in an anticipated attempt to reduce the adhesion, we have tested masks with a superhydrophobic coating. It is found that a superhydrophobic coating may not be the best choice for a regular mask as it can give rise to a number of smaller daughter droplets and thus can linger in air for longer time and can contribute to the transmission of potential viral loads.
\end{abstract}

\section{Introduction}

The coronavirus pandemic has affected the world severely, claiming many lives and rendering a huge population under risk of getting contracted easily. It is caused by a specific strain of coronavirus (SARS$\mathrm{CoV}-2$ ) which is believed to be transmitted through aerosol that is laden with the virus. ${ }^{1}$ In absence of therapeutics and wide spread immunisation, a preventive strategy is the best option at present to mitigate this public health crisis. In this regard, face covering is the most effective way in preventing the COVID19 pandemic, according to the World Health Organization (WHO) ${ }^{2}$ and among which a simple face mask is oldest and time tested method of first line defense. ${ }^{3}$ Among different types and materials, 3-ply surgical mask based on polypropylene is the most used one. They have the highest filter quality among all other daily used masks. ${ }^{4}$ Though extensive studies have been conducted on the efficacy the masks (ability to seal air flow) in terms of blowing out air through them to understand the hydrodynamics related to the air flow produced by the individual who is wearing the mask, ${ }^{5-9}$ the reverse case of the aerosol interaction with the mask (i.e., interaction of droplets on to the mask from external environment) has not gained much 
momentum, despite its critical role in the transmission of the virus.

A face mask is a porous structure that allows fair breathing but at the same time, prevents the disease-causing pathogens to pass through the mask and enter the respiratory track of an individual. Though highly efficient masks like N95 is the best option however this can cause discomfort in normal breathing and cannot be used for a long time due to a very small pore size $(0.3 \mu \mathrm{m})$. Further, the widespread use of such masks will affect their supply to the healthcare workers who have a better probability of encountering large quantity of the viral load and thus require the most effective defense first. WHO has also suggested the use of home- made cloth masks (termed as 'non-medical') with multiple layers as a ready-to-use alternative to disposable masks and are cost-effective and affect environment less harmfully than the polymeric based masks which leaves a larger carbon footprint. ${ }^{10}$

The basic mechanism of a mask in protecting from viruses is that the aerosol droplets containing viruses are captured in the porous structures of the mask and do not allow them to be inhaled into the respiratory system. These droplets can be in the range of $0.1-1 \mathrm{~mm}$ in size. ${ }^{11}$ and thus a mask with smaller pore size should be employed and is more effective when they are in multiple layers, which further reduces the probability of any droplet getting through. The droplet behavior depends on the fundamental interactions of the fluid interface with the porous structures formed by the individual strands of the mask fibres. An uneven structured surface can significantly affect the contact time of the droplet during impact ${ }^{12}$ and therefore any mask should be tested for adhesion and impact, which are the physical mechanisms in place in a real-scenario of a virus-laden aerosol particle. There are numerous attempts to enhance the capability of trapping particles in a mask like chemical treatment ${ }^{13}$ and triboelectric enhancement for a charge based repellence. ${ }^{4}$ As any enhancement in the repellency and non-attachment of the droplets is a positive aspect of the mask without affecting its breathability, one of the obvious propositions to repel any droplet is to have superhydrophobic coatings over the masks, which can repel the droplets owing to a low surface energy. Such scenarios have been studied extensively for flat surfaces for various applications including self- cleaning, ${ }^{14-16}$ anti-icing ${ }^{17,18}$ and thermal applications ${ }^{19,20}$ to name a few. There are also extensive studies on the impact of droplets on superhydrophobic surfaces, both chemically treated and with micro/nano structures. ${ }^{12,20-23}$ A recent textile based study has shown that the superhydrophobic treatment may not ensure a repellency for a practical situation like rain. ${ }^{24}$ However, there are no studies currently available on masks coated with such repellent materials to the best of our knowledge.

In this present work, we explore the wetting, impact and adhesion of droplets over the commonly used 3-ply surgical face mask. A high-speed imaging is carried out to study the impact dynamics and the droplet formation, which are critical in understating the formation of aerosols, which further directly affect the spreading capability of the virus laden droplets. Both sides of the masks, the front and back are tested 
separately regarding their effectiveness. A regular water droplet and a droplet laden with microbe (Escherichia coli (E. coli)) are considered. Further, the superhydrophobic coating, which under normal circumstances will enhance the repellent behavior of the mask, is also applied on the mask to understand the interfacial phenomena compared to the one without any coating.

\section{Experimental}

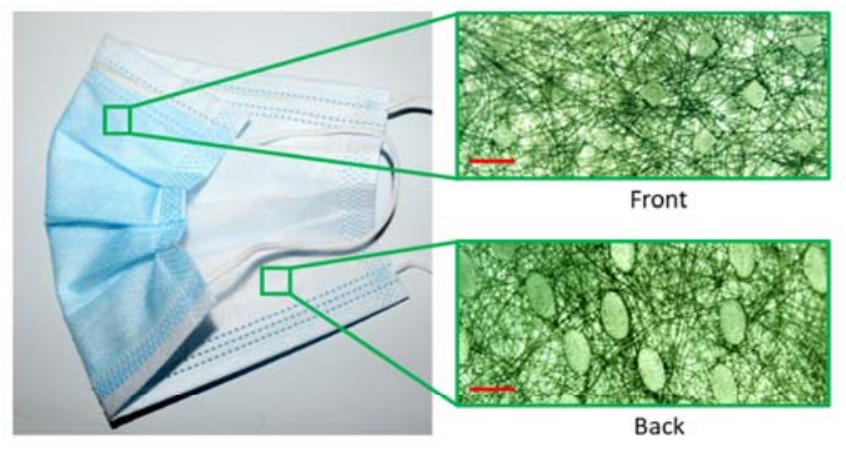

(a)

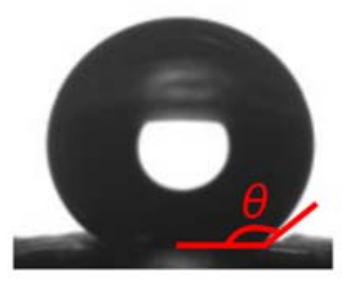

(b)

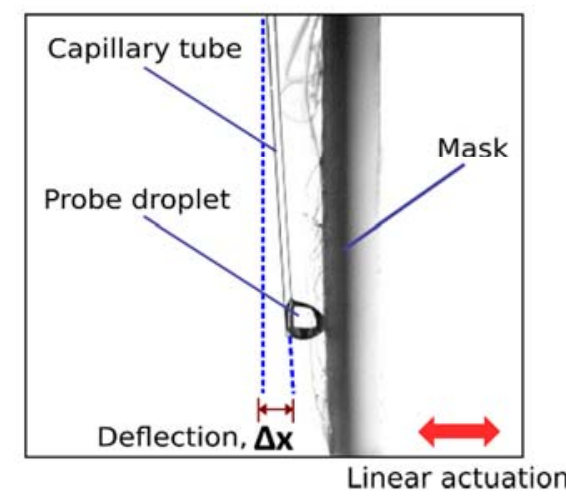

(c)

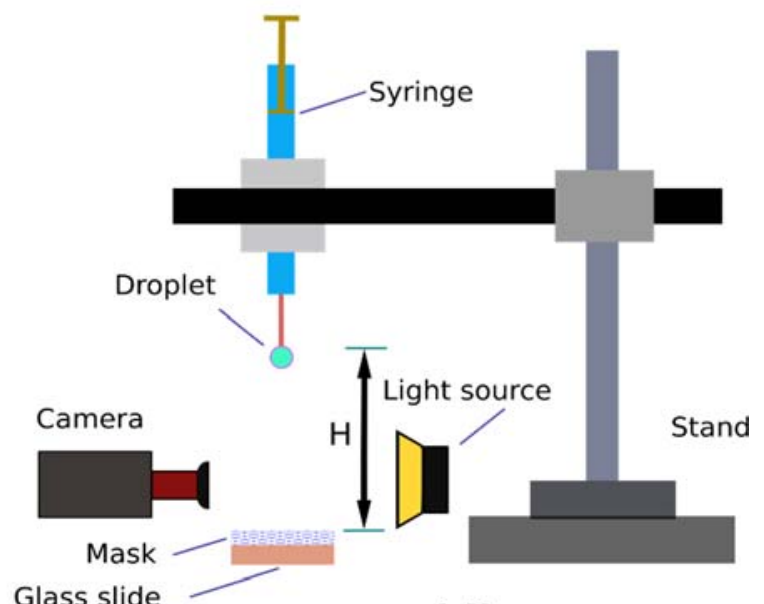

(d)

Figure 1. (a)The front and the back side of a 3-ply face mask with zoomed in portions showing individual fibre strands and periodically appearing fused elements of the non-woven fabric. Scale bar represents $1 \mathrm{~mm}$ (b) A static contact angle $(\theta)$ of the test droplet (DI water) on an uncoated mask, represented as a sessile drop in contact with the mask (c) The adhesion measurement apparatus where the deflection $(\Delta x)$ is tracked with respect to time (d) The impact measurement setup in which the droplet is dispensed from a height $\mathrm{H}$.

We used disposable 3-ply face masks (Intermask, Singapore) made of polypropylene non-woven fabric. Both front and back sides are shown in Figure 1(a) with microscopic images showing the intermittent patches for holding the fibers together. For the superhydrophobic coating, we used a fluorinated coating in liquid form (Glass Protect-SH, SiO2 Innovation Labs, Canada). To treat the mask with a superhydrophobic coating, we dipped it in the coating solution for $5 \mathrm{~min}$ and subsequently heated inside a hot air oven for $1 \mathrm{hr}$ at $60{ }^{\circ} \mathrm{C}$ for drying. Uncoated masks are used as it is. Both masks are cut and attached horizontally over a glass slide of size $1 \mathrm{~cm} \times 1 \mathrm{~cm}$ using a scotch tape on the four sides. As test 
liquids, deionized water (DI) and an overnight cultured bacterium (E. coli) in Lauryl Trypose broth without any dilution is used. Conventional goniometry was used to perform the static contact angle measurements (DSA 30, Krüss USA) as shown in Figure 1(b). For contact angle hysteresis (CAH) measurement, the test liquid was slowly dispensed on the test surface at a flowrate of $0.1 \mu \mathrm{L} / \mathrm{min}$, increasing the volume of the droplet, and the advancing contact angle $\left(\theta_{a d v}\right)$ is estimated once the contact line starts moving. Further, during the retraction of the test fluid, the receding contact angle $\left(\theta_{\text {rec }}\right)$ is estimated at the first instant of the contact line motion. ${ }^{25}$ Then the difference of the two angles is evaluated as $\mathrm{CAH}=\theta_{a d v}-\theta_{\text {rec }}$. Adhesion force $\left(F_{a d h}\right)$ is measured using an in-house developed cantilever based sensor whose mechanism is described elsewhere. ${ }^{26}$ Briefly, the droplet is dispensed at the tip of a polymeric capillary (diameter $=360 \mathrm{~mm}$, length $=12 \mathrm{~mm}$, Paradigm Optics Inc, WA, USA) as shown in Figure 1(c). Using a motorized linear actuation with a velocity of $500 \mu \mathrm{m} / \mathrm{s}$, (Zaber Technologies Inc., BC, Canada), the capillary with the droplet attached is brought towards a vertically held sample and then detached after a brief contact of $10 \mathrm{~s}$, the deflection $(\Delta x)$ is measured from the camera image (Photron FASTCAM Mini) (see supporting information, Video S1). From the simple linear force-displacement relation, $F=k \Delta x$ (where $k$, the spring constant of the capillary, which is measured separately in a calibration experiment, is $19.3 \mathrm{mN} / \mathrm{m}$ - for details, please see our earlier publications ${ }^{26,27}$ ), the adhesion force $F_{a d h}$ can be evaluated as the force at maximum displacement $\left(F_{a d h}=k \Delta x_{\max }\right)$. For impact, the droplets are dispensed from a height $\mathrm{H}$ as shown in Figure 1(d) and captured using a high-speed imaging at 6400 frames per second with a strong LED backlight. The droplets used here have diameter $d=1.5 \pm 0.23 \mathrm{~mm}$ and were dispensed at a varying height of $\mathrm{H}=5 \mathrm{~cm}, 10 \mathrm{~cm}$, and $15 \mathrm{~cm}$, respectively. This results in an impact velocity $(\mathrm{u})$ on the tested surfaces, which varies with the height $\mathrm{H}$ as $0.99 \mathrm{~m} / \mathrm{s}, 1.4$ $\mathrm{m} / \mathrm{s}$, and $1.71 \mathrm{~m} / \mathrm{s}$, respectively. One can define a resulting impact Weber number (relative strength of inertial force over surface tension force $), W e=\frac{\rho u^{2} d}{\gamma}$ where $\rho\left(=1000 \mathrm{~kg} / \mathrm{m}^{3}\right.$ for DI water; $=1006 \mathrm{~kg} / \mathrm{m}^{3}$ for $E$. coli solution $)$ is the density and $\gamma(=72.6 \pm 2.7 \mathrm{mN} / \mathrm{m}$ for DI water; $=57.8 \pm 3 \mathrm{mN} / \mathrm{m}$ for $E$. coli solution) is the surface tension of the liquid. The value of $W e$ varies based on the impact velocity and the working fluid used. Therefore, for DI water, the values of $W e$ used here are 20.41, 40.8, and 61.25; whereas for E. coli solution they are $25.59,51.17$, and 76.76, respectively. Further image analysis is carried out in ImageJ (National Institutes of Health, USA) and Matlab (Mathworks, Natick, MA, USA) to estimate timestamps and the corresponding droplet geometry. Each measurement was taken at least three times, at three different locations of the face mask (front and back for both coated and uncoated surfaces). The ambient temperature and relative humidity during the experiments were recorded as 21.5 $\pm 0.5^{\circ} \mathrm{C}$ and $52 \pm 4 \%$ respectively. 


\section{Results \& Discussion}

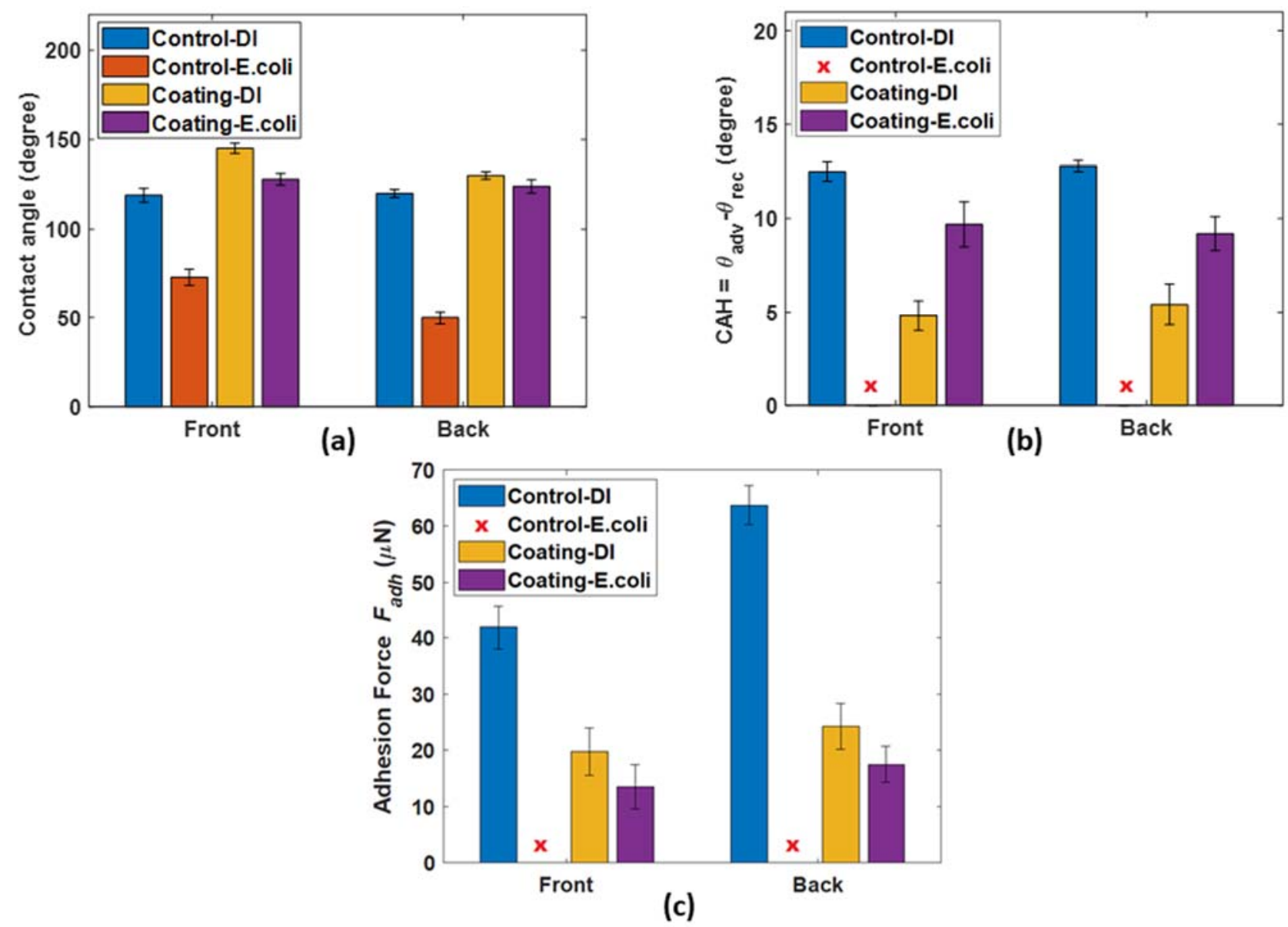

Figure 2. (a) Static contact angle $(\theta)$ (b) Contact angle hysteresis $(\mathrm{CAH})(\mathrm{c})$ Adhesion force $\left(F_{\text {adh }}\right)$ values for the front and back side of the mask without coating (control) and with coating for DI water and E. coli laden droplet, respectively. Note that "red" cross indicates the values that cannot be measured for $E$. coli laden droplet on uncoated mask.

Figure 2(a) shows the static contact angle values for a sessile droplet dispensed on both sides (front and back) of the face mask. The superhydrophobic coating affected the mask similar to a flat surface, by lowering the surface energy, resulting in a high contact angle with water and E.coli. The effect of E.coli is perceived as the reduction of the overall static contact angle, more pronounced for uncoated one (control) where a $38 \%$ reduction is observed than the coated one where only $11 \%$ is observed. E.coli penetrated the first layer of the mask where the droplet is dispensed for the uncoated mask, thus the contact angle measured here is affected by the wettability of the middle layer also. Such a penetration was not observed for any other cases, limiting the interaction of the droplet on the top layer only. Even though not significant, the contact angles were smaller for the back side of the mask than the front, inferring only a marginal advantage for the front side being exposed. Also, the lowest contact angle among all the cases was observed for the back side with E.coli. Although useful in understanding the wettability of the 
surface, static contact angle provides minimal information regarding the mobility of the droplet over the surface, which is important for superhydrophobic surfaces. In order to account for the dynamic wetting signature, we have also carried out the contact angle hysteresis for all the cases as shown in Figure 2(b). Due to the noticeable penetration of the test liquid while dispensing on the mask, a reliable measurement was not possible for the bacteria laden droplet for the uncoated case. For the rest of the cases, similar wetting behavior for both the front and the back sides of the mask was observed among which the water droplet on the coated mask showed the least hysteresis.

Figure 2(c) shows $F_{\text {adh }}$ for both sides of the masks for all the cases considered. During the adhesion measurement, the individual fibres of the mask affect the droplet detachment from the surface, creating more pinning sites compared to a flat surface. Similar to the case of contact angle measurements of the bacteria laden droplet for the uncoated mask, penetration of the test liquid into the mask resulted in strong pinning and eventual detachment of the droplet from the cantilever and thus could not be reported. The uncoated mask has the highest $F_{a d h}$ and the superhydrophobic coating has reduced $F_{a d h}$ for both sides, corroborating the results from the contact angle measurements. Further, it was also observed that $F_{\text {adh }}$ was lesser for the front than the back for both DI and E.coli, emphasizing a marginal advantage of the front side in repelling the droplets. In our recent work, ${ }^{27}$ we have conducted a detailed measurement of the adhesion force experienced by bacteria laden droplets on flat superhydrophobic surfaces (i.e., coated glass surfaces) where we observed that E.coli laden drops have a reduced $F_{a d h}$ compare to DI drops, implying the direct effect of the bacterial motility. The same trend is observed here in case of coated masks where E.coli laden droplets have a lesser adhesion compared to the DI water droplets. This shows that the bacterial motility over a porous surface like mask is similar to a flat surface with a repellent coating even though there are more pinning sites for the former test surface (mask) compared to the latter (glass). As observed from Figure 1(c), the tiny fibres of the mask will protrude from the surface due to the non-woven nature of the polypropylene fibres that are used during the fabrication of both outer and inner layers of a 3-ply mask. They will act as additional pinning points on the surface, thereby increasing the adhesion compared to a comparably flatter surface of the same material for instance, the intermediate layer of the 3-ply mask, which is made from the same polymer using melt-blown technique with a higher density compared to other layers.

Figure 3 shows the behavior of droplets on impact on the front surface of the face mask. For uncoated mask, with DI water, there is a crown formation and eventually settles down as a sessile droplet without any splashing (Fig. 3(a)) and for E.coli droplet, there is a less prominent crown formation (Fig. 3(b)). For the coated mask, it bounces with remarkable elastic behavior as observed for nonporous and flat superhydrophobic surfaces. ${ }^{21}$ Here, the droplet is completely detached after the initial 


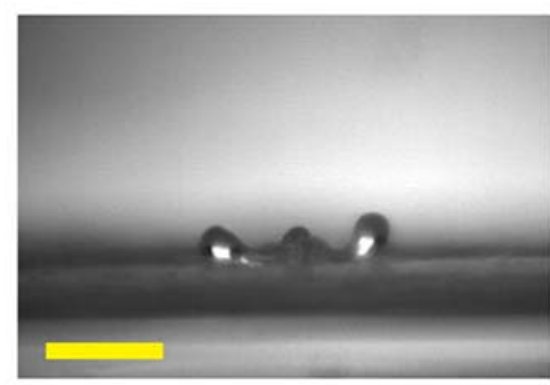

(a)

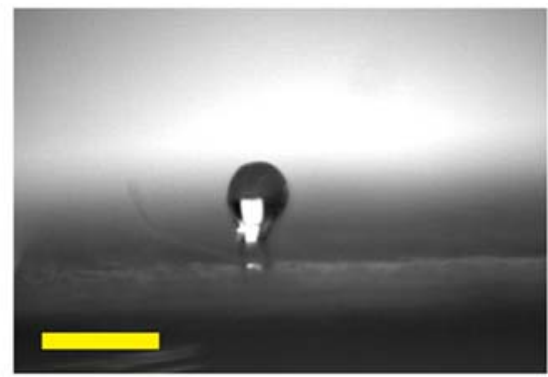

(c)

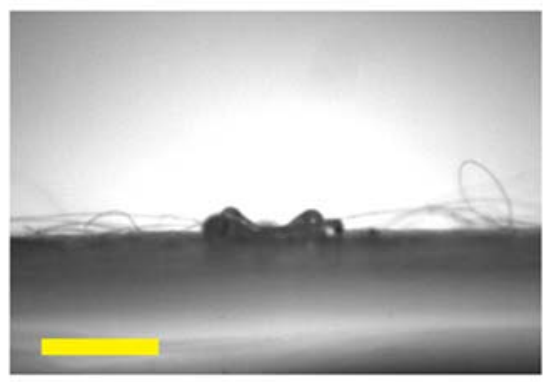

(b)

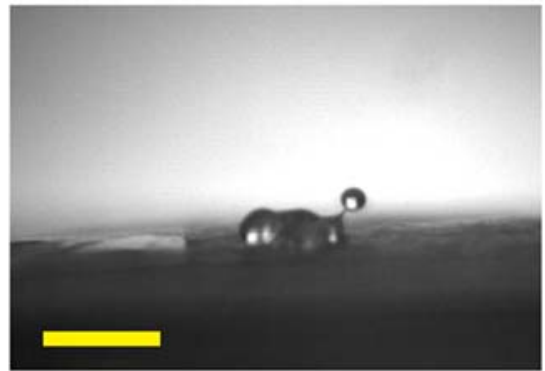

(d)

Figure 3. The impact of droplets over masks on the front side from a height $\mathrm{H}=5 \mathrm{~cm}$ for (a) uncoated mask with DI drop (control) ( $W e=20.41)(b)$ uncoated mask with E.coli drop (control) $(W e=25.59)$ (c) coated mask with DI drop ( $W e=$ 20.41) (d) coated mask with E.coli drop $(W e=25.59)$. Scale bar represents $1 \mathrm{~mm}$.

impact, bounced back and then reattached on the second impact (Figure 3(c)). For the case of coated mask with E.coli, there is a splashing of the droplet, forming daughter droplets and scattering in a direction away from the original droplet (Figure 3(d)). The results here cannot be directly compared against wetting studies on textiles ${ }^{24}$ with the associated scaling relationships for the penetration (as a function of $W e$ and the mesh size) as the masks are multi-layered and has a solid support from behind, which corresponds to the normal usage of masks over the face.

It is to be noted that the impact dynamics is also a function of the height $\mathrm{H}$ from which the droplet is dispensed on the target surfaces. The daughter droplet formation is found to be a function of the $W e$, implying the significance of the inertial forces in creating the additional interfacial area as the number of daughter droplets are formed from a single droplet. Even with the DI water, for moderately low $W e$, i.e., for $\mathrm{H} \leq 10 \mathrm{~cm}$ in our study, as show in Figures 4 (a) and (b), there is a crown formation of the droplet and subsequent wetting but without any splashing on the uncoated mask surface. However, at a relatively higher $W e=61.25$ (corresponding to $\mathrm{H}=15 \mathrm{~cm}$ ), as shown in Figure. 4 (c), we do observe splashing to occur on the front side of the uncoated masks before wetting the surface through individual daughter droplets. In the case of impact of DI water droplet on coated masks, moderately low We resulted 
$W e=20.41$

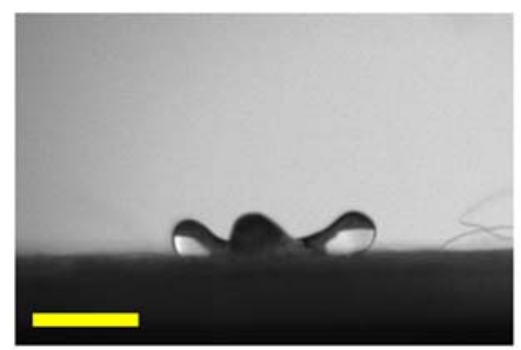

(a)

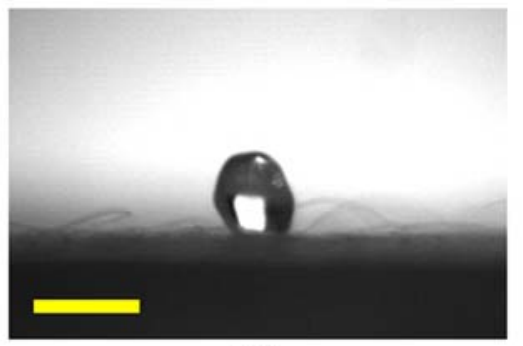

(d)
$W e=40.8$

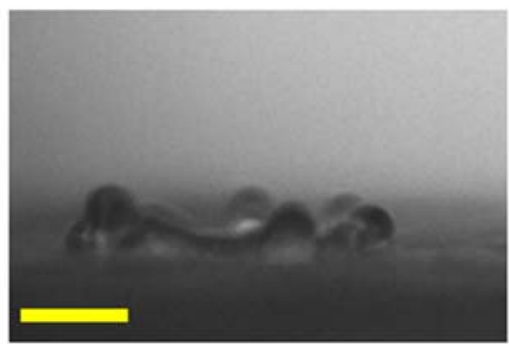

(b)

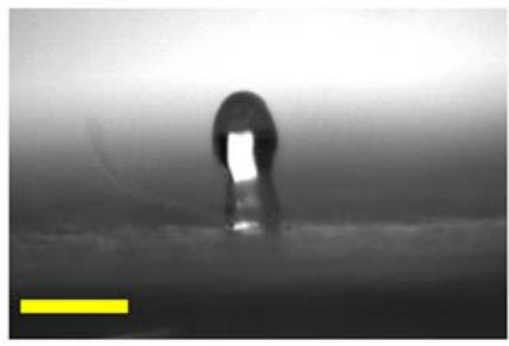

(e)
$W e=61.25$

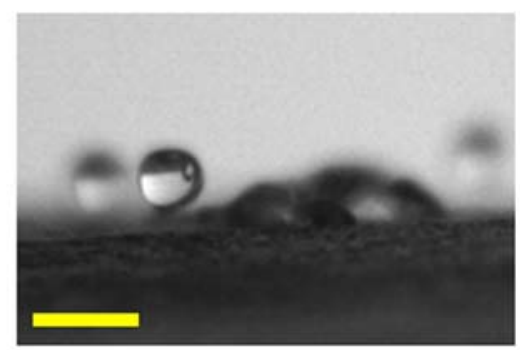

(c)

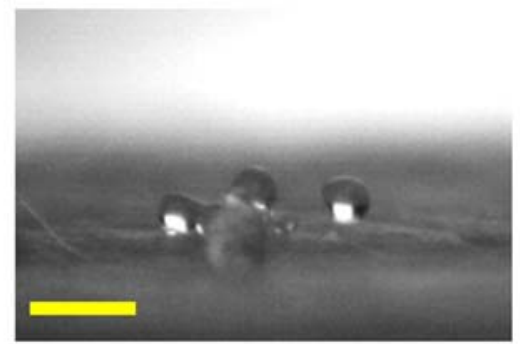

(f)

Figure 4. Impact characteristics of DI drop on front side of the mask at the same instant for (a-c) uncoated (control) mask and (d-f) coated mask for $W e=20.41(\mathrm{H}=5 \mathrm{~cm}), W e=40.8(\mathrm{H}=10 \mathrm{~cm})$ and $W e=61.25(\mathrm{H}=15 \mathrm{~cm})$. Scale bar represents $1 \mathrm{~mm}$.

in the jumping of the droplets without wetting the mask and subsequent elongation due to the elastic recoiling as shown in Figures 4 (d) and (e) with a higher elongation for $W e=40.8$. However, at high We $(=61.25)$, splashing behavior similar to the uncoated mask was observed as shown in Figure 4 (f). For front and back surfaces of the face mask, the impact behavior was similar for all the cases, which is the same as that observed for the contact angle measurements. Structurally, the difference between the front and the back is in the fused elements of the non-woven fabric that holds the fibres together as shown in Figure 1(a). The back side has a more unevenly placed fused elements than the front, but it is not sufficient to create a dramatic difference in the wetting and impact signatures, thus scientifically ruling out the differences in the usage of either sides to prevent an aerosol impact. In terms of the coating, the observations suggest that the coating can give rise to daughter droplets easily compared to an uncoated mask and thus aid in the airborne transmission as smaller droplet tend to stay in air, defying gravity and can be transported by the air currents to long distances before settling down. The splashing over the superhydrophobic coated masks can occasionally produce droplets of size $\sim 100 \mu \mathrm{m}$ which is the critical size below which they fail to settle by gravity. ${ }^{28}$

One other aspect that is relevant to this problem is the stretched configuration of the mask. It will have an effect on the droplet impact characteristics, acting like a stretched membrane. For a mask held with a lesser lateral tension over the glass slide than what is used here in the present study, the kinetic energy of the impacting droplet is absorbed into the mask fibres on the top layer and the two other 
supporting layers below, thus reducing the degree of splashing and eventual formation of the daughter droplets ${ }^{29}$ (see supporting information, Video S2). Here, deformed interface of the droplet is limited itself to the crown formation, which otherwise would have splashed into daughter droplets if the mask did not cushioned itself to accommodate the impact. This measure of the stretching could be correlated to how tightly we fix our masks around our face. In this context, a mask without a hard back support will reduce the formation of the daughter droplets. Further in terms of wearing the mask, the orientation of the mask with respect to the impinging droplet is a critical factor that dictates the impact dynamics. For the case of complete bouncing upon impact (Figure 3c) on coated mask with lesser contact angle hysteresis $\left(\sim 5^{0}\right)$ compared to uncoated ones, the droplet will follow the conventional pathway of spreading to a maximum diameter, recoiling and leaving the surface without adhering to the mask. For the case in which splashing and daughter droplet formation was observed (Figure 3d), a change in the angle of impact will alter the normal reaction force $(\mathrm{W} \cos \alpha$, where $\mathrm{W}$ is the weight of the droplet and $\alpha$ is the angle of inclination of the mask with the horizontal) and therefore the trajectory of the daughter droplets post impact. Another aspect which is important to this study is the droplet size. The usual size in a cough/sneeze aerosol is much smaller than what is studied here. But there exists still a probability of large droplets of the order of $\mathrm{mm}$ in extreme cases and often such large droplets are used in experiments, such as microbead laden droplets to model cough/sneeze. ${ }^{11,30,31}$ We would also like to mention the importance of humidity as it can significantly affect the wetting signature of any dropletsurface combination, measured in air. Through this work, we aimed to clarify the common conception about which side to use (front or back) in the case of a 3-ply mask and focused on the simplest case of wetting in ambient air medium with room temperature conditions. Clearly, whichever side is used that comes in contact with the face, the wetting will be affected by the highly humid environment that is created within the space between the nose and the mask due to the moisture from exhaled air (Typically, this relative humidity value can be as high as $90 \%) .{ }^{32}$ A detailed study is required to elucidate the effect of ambient humidity on the wetting signature of microbe-laden droplets on masks. In summary, this study points out the relevance of superhydrophobic coatings as a modifier to existing masks and provides some clue on how effective they are when applied on both sides of the mask (front and back), with respect a microbial droplet adhesion.

\section{Conclusion}

We have carried out a detailed investigation of droplet interaction with the polypropylene 3-ply face mask that is most commonly used during the global pandemic of COVID-19. We have performed wetting (both static and dynamic), adhesion and impact characteristics for masks treated with a superhydrophobic coating aimed to repel incoming droplets and compared the results against uncoated masks. Such 
superhydrophobic coatings are often used in designer cloth masks to give a glossy finish. Both water droplets and droplets laden with bacteria are used as the test fluid. There was no significant difference in the impact dynamics between the front and the back sides of the masks, but the contact angle and adhesion force measurements showed a marginal advantage for the front side of the mask in terms of their repellency towards droplets. We conclude that the superhydrophobic coating, which may be effective in repelling statically dispensed droplets, will cause the formation of daughter droplets upon impact easily compared to an uncoated mask and thus may be detrimental in a practical scenario of airborne viral spreading during a pandemic including COVID-19.

\section{Supporting information}

Video of adhesion measurement (Video S1)

Video of impact over an uncoated mask (Video S2)

\section{Acknowledgements}

The authors would like to thank $\mathrm{SiO} 2$ Innovation Labs for graciously providing the superhydrophobic coatings used in this work. This work was supported by S.K.M.'s Alliance Grant ALLRP 551068-20 from the Natural Sciences and Engineering Research Council (NSERC) of Canada, Mitacs Accelerate, and University of Waterloo Start-up grant.

\section{References}

(1) Wang, C.; Horby, P. W.; Hayden, F. G.; Gao, G. F. A Novel Coronavirus Outbreak of Global Health Concern. Lancet 2020, 395 (10223), 470-473.

(2) Covid-, W. H. O. Advice on the Use of Masks in the Context of COVID-19. Who 2020, No. April, $1-5$.

(3) Weaver, G. H. Droplet Infection and Its Prevention by the Face Mask. J. Infect. Dis. 1919, 24 (3), 218-230.

(4) Zhao, M.; Liao, L.; Xiao, W.; Yu, X.; Wang, H.; Wang, Q.; Lin, Y. L.; Kilinc-Balci, F. S.; Price, A.; Chu, L.; Chu, M. C.; Chu, S.; Cui, Y. Household Materials Selection for Homemade Cloth Face Coverings and Their Filtration Efficiency Enhancement with Triboelectric Charging. Nano Lett. 2020, 20 (7), 5544-5552.

(5) Verma, S.; Dhanak, M.; Frankenfield, J. Visualizing Droplet Dispersal for Face Shields and Masks with Exhalation Valves. Phys. Fluids 2020, 32 (9), 091701.

(6) Bahl, P.; Bhattacharjee, S.; de Silva, C.; Chughtai, A. A.; Doolan, C.; MacIntyre, C. R. Face Coverings and Mask to Minimise Droplet Dispersion and Aerosolisation: A Video Case Study. Thorax 2020, 75 (11), 1024-1025. https://doi.org/10.1136/thoraxjnl-2020-215748.

(7) Dbouk, T.; Drikakis, D. On Respiratory Droplets and Face Masks. Phys. Fluids 2020, 32 (6), 063303.

(8) Bourouiba, L. Turbulent Gas Clouds and Respiratory Pathogen Emissions. JAMA 2020, 323 (18), 1837-1838.

(9) Bourouiba, L.; Dehandschoewercker, E.; Bush, J. W. M. Violent Expiratory Events: On Coughing and Sneezing. J. Fluid Mech. 2014, 745, 537-563.

(10) B., O. D.; Mona, S. Environmental Hygiene for COVID-19: It's All About the Mask. J. Environ. 
Eng. 2020, 146 (12), 1820004.

(11) Han, Z. Y.; Weng, W. G.; Huang, Q. Y. Characterizations of Particle Size Distribution of the Droplets Exhaled by Sneeze. J. R. Soc. Interface 2013, 10 (88), 20130560.

(12) Liu, Y.; Moevius, L.; Xu, X.; Qian, T.; Yeomans, J. M.; Wang, Z. Pancake Bouncing on Superhydrophobic Surfaces. Nat. Phys. 2014, 10 (7), 515-519.

(13) Ray, S. S.; Park, Y.-I.; Park, H.; Nam, S.-E.; Kim, I.-C.; Kwon, Y.-N. Surface Innovation to Enhance Anti-Droplet and Hydrophobic Behavior of Breathable Compressed-Polyurethane Masks. Environ. Technol. Innov. 2020, 20, 101093.

(14) Blossey, R. Self-Cleaning Surfaces_-Virtual Realities. Nat. Mater. 2003, 2 (5), 301-306.

(15) Tuteja, A.; Choi, W.; Ma, M.; Mabry, J. M.; Mazzella, S. A.; Rutledge, G. C.; McKinley, G. H.; Cohen, R. E. Designing Superoleophobic Surfaces. Science (80-. ). 2007, 318 (5856), 1618-1622.

(16) Deng, X.; Mammen, L.; Butt, H. J.; Vollmer, D. Candle Soot as a Template for a Transparent Robust Superamphiphobic Coating. Science (80-. ). 2012, 335 (6064), 67-70.

(17) Stone, H. A. Ice-Phobic Surfaces That Are Wet. ACS Nano 2012, 6 (8), 6536-6540.

(18) Vakarelski, I. U.; Patankar, N. A.; Marston, J. O.; Chan, D. Y. C.; Thoroddsen, S. T. Stabilization of Leidenfrost Vapour Layer by Textured Superhydrophobic Surfaces. Nature 2012, 489 (7415), 274-277.

(19) Chen, X.; Wu, J.; Ma, R.; Hua, M.; Koratkar, N.; Yao, S.; Wang, Z. Nanograssed Micropyramidal Architectures for Continuous Dropwise Condensation. Adv. Funct. Mater. 2011, 21 (24), 46174623.

(20) Richard, D.; Clanet, C.; Quéré, D. Contact Time of a Bouncing Drop. Nature 2002, 417 (6891), 811-811.

(21) Tsai, P.; Pacheco, S.; Pirat, C.; Lefferts, L.; Lohse, D. Drop Impact upon Micro- and Nanostructured Superhydrophobic Surfaces. Langmuir 2009, 25 (20), 12293-12298.

(22) Iqbal, R.; Majhy, B.; Sen, A. K. Facile Fabrication and Characterization of a PDMS-Derived Candle Soot Coated Stable Biocompatible Superhydrophobic and Superhemophobic Surface. ACS Appl. Mater. Interfaces 2017, 9 (36), 31170-31180.

(23) Majhy, B.; Iqbal, R.; Sen, A. K. Facile Fabrication and Mechanistic Understanding of a Transparent Reversible Superhydrophobic--Superhydrophilic Surface. Sci. Rep. 2018, 8 (1), 111.

(24) Zhang, G.; Quetzeri-Santiago, M. A.; Stone, C. A.; Botto, L.; Castrejón-Pita, J. R. Droplet Impact Dynamics on Textiles. Soft Matter 2018, 14 (40), 8182-8190.

(25) Huhtamäki, T.; Tian, X.; Korhonen, J. T.; Ras, R. H. A. Surface-Wetting Characterization Using Contact-Angle Measurements. Nat. Protoc. 2018, 13 (7), 1521-1538.

(26) Melayil, K. R.; Misra, S.; Mitra, S. K. Friction and Adhesion of Microparticle Suspensions on Repellent Surfaces. Langmuir 2020, 36 (45), 13689-13697.

(27) Melayil, K. R.; Misra, S.; Mitra, S. K. Microparticle Suspensions and Bacteria-Laden Droplets: Are They the Same in Terms of Wetting Signature? Langmuir 2021, 37 (4), 1588 - 1595.

(31) Wei, J.; Li, Y. Enhanced Spread of Expiratory Droplets by Turbulence in a Cough Jet. Build. Environ. 2015, 93 (P2), 86-96.

(32) Mansour, E.; Vishinkin, R.; Rihet, S.; Saliba, W.; Fish, F.; Sarfati, P.; Haick, H. Measurement of Temperature and Relative Humidity in Exhaled Breath. Sensors Actuators, B Chem. 2020, 304 (October 2019), 127371. 


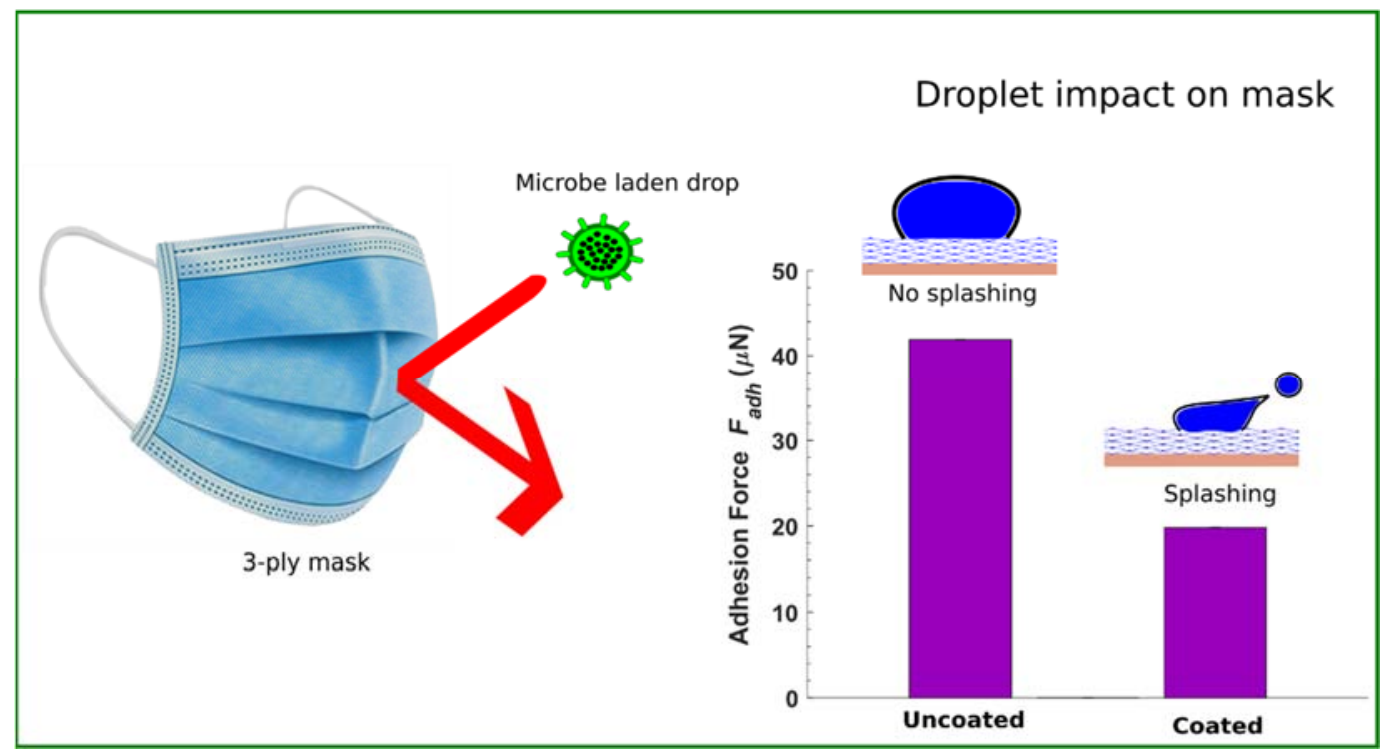

TOC Graphic 